\title{
Front Matter: Volume 10310
}

, "Front Matter: Volume 10310," Proc. SPIE 10310, Technology of Proximal Probe Lithography, 1031001 (3 October 1993); doi: 10.1117/12.2283725

SPIE Event: SPIE Institutes for Advanced Optical Technologies 10, 1993, SPIE. Bellingham, WA, United States 


\section{Table of Contents}

ix List of Contributors

\section{INTRODUCTION}

3 Technology of proximal probe lithography: an overview

J. A. Dagata, National Institute of Standards and Technology;

C. R. K. Marrian, Naval Research Lab.

\section{PART I. NANOLITHOGRAPHY}

16 Principles and techniques of STM lithography

M. A. McCord, IBM Corp.; R. F. W. Pease, Stanford Univ.

33 Role of scanning probe microscopes in the development of nanoelectric devices

A. Majumdar, S. M. Lindsay, Arizona State Univ.

58 Low voltage e-beam lithography with the STM

C. R. K. Marrian, E. A. Dobisz, Naval Research Lab.; J. A. Dagata, National Institute of Standards and Technology

74 Nanolithography and atomic manipulation on silicon surfaces by STM F. Grey, A. Kobayashi, H. Uchida, D. H. Huang, M. Aono, Aono Atomcraft Project (Japan)

100 Scanning tunneling microscope-based fabrication and characterization on passivated semiconductor surfaces

J. A. Dagata, J. Schneir, National Institute of Standards and Technology

111 Scanning tunneling microscope-based nanolithography for electronic device fabrication

J. W. Lyding, R. T. Brockenbrough, P. Fay, J. R. Tucker, K. Hess, Univ. of Illinois; T. K. Higman, Univ. of Minnesota

127 Arrayed lithography using STM-based microcolumns

T. H. P. Chang, IBM T.J. Watson Research Ctr.; L. P. Muray, Cornell Univ.; U. Staufer, Univ. of Basel (Switzerland); M. A. McCord, D. P. Kern, IBM T.J. Watson Research Ctr.

\section{PART II. FABRICATION}

162 Nanofabrication by scanning probe instruments: methods, potential applications, and key issues

R. Wiesendanger, Univ. of Basel (Switzerland)

188 Direct writing of metallic nanostructures with the scanning tunneling microscope

A. L. de Lozanne, W. F. Smith, E. E. Ehrichs, Univ. of Texas/Austin Technology of Proximal Probe Lithography, edited by Christie R. K. Marrian, Proc. of SPIE Vol. 10310 (Vol. IS10), 1031001 - ( ) (1993) 2017 SPIE CCC code: $0277-786 X / 17 / \$ 18 \cdot$ doi: $10.1117 / 12.2283725$ 
200 Fabrication of nanometer-scale structures

M. H. Nayfeh, Univ. of Illinois/Urbana-Champaign

218 Modification and manipulation of layered materials using scanned probe microscopies

C. M. Lieber, Harvard Univ.

234 Atomic force microscopy experimentation at surfaces: hardness, wear, and lithographic applications

T. A. Jung, Univ. of Basel (Switzerland) and Paul Scherrer Institute (Switzerland); A. Moser, Univ. of Basel (Switzerland); M. T. Gale, Paul Scherrer Institute (Switzerland); H. J. Hug, U. D. Schwarz, Univ. of Basel (Switzerland)

268 Fabrication and characterization using scanned nanoprobes G. C. Wetsel Jr., S. E. McBride, H. M. Marchman, Univ. of Texas/Dallas

289 Ballistic electron emission microscopy: from electron transport physics to nanoscale materials science

H. D. Hallen, AT\&T Bell Labs.

\section{PART III. METROLOGY}

322 Generating and measuring displacements up to $0.1 \mathrm{~m}$ to an accuracy of $0.1 \mathrm{~nm}$ : Is it possible?

E. C. Teague, National Institute of Standards and Technology

364 Metrology with scanning probe microscopes

J. E. Griffith, AT\&T Bell Labs.; D. A. Grigg, Digital Instruments;

G. P. Kochanski, M. J. Vasile, AT\&T Bell Labs.; P. E. Russell, North Carolina State Univ.

390 Metrology applications of scanning probe microscopes

L. A. Files-Sesler, J. N. Randall, F. G. Celii, Texas Instruments Inc. 


\section{List of Contributors}

M. Aono

Aono Atomcraft Project

Exploratory Research for Advanced Technology

Japan Research Development Corp.

Tokodai 5-9-9

Tsukuba, Ibaraki 300-26, Japan

Roger T. Brockenbrough

Univ. of Illnois

Dept. of Electrical and Computer

Engineering

Urbana, IL 61801, USA

Francis G. Celii

Texas Instruments Inc.

MS 147

P.O. Box 655936

Dallas, TX 75265-5936, USA

T. H. Chang

IBM T.J. Watson Research Ctr.

Yorktown Heights, NY 10598, USA

John A. Dagata

National Institute of Standards and

Technology

Bldg. 220, Rm. A107

Gaithersburg, MD 20899-0001, USA

A. L. de Lozanne

Univ. of Texas/Austin

Physics Dept.

Austin, TX 78712-1020, USA

Elizabeth A. Dobisz

Naval Research Lab.

Electronics Science \& Technology Div.

Code 6864

4555 Overlook Dr.

Washington, DC 20375-5347, USA

E. E. Ehrichs

Univ. of Texas

Dept. of Physics

Austin, TX 78712-1081, USA

Patrick Fay

Univ. of Illinois

Dept. of Electrical and Computer

Engineering

Urbana, IL 61801, USA
Leigh A. Files-Sesler

Texas Instruments Inc.

Central Research Labs.

MS 134

13588 N. Central Expwy.

Dallas, TX 75243-1108, USA

Michael T. Gale

Paul Scherrer Institute

Optics Group

Badenerstrasse 569

CH-8048 Zürich, Switzerland

François Grey

Aono Atomcraft Project

Exploratory Research for Advanced

Technology

Japan Research Development Corp.

Tokodai 5-9-9

Tsukuba, Ibaraki 300-26, Japan

Joseph E. Griffith

AT\&T Bell Labs.

Rm. 6F-225

P.O. Box 636

Murray Hill, NJ 07974-0636, USA

David A. Grigg

Digital Instruments

520 E. Montecito

Santa Barbara, CA 93103-3252

Hans D. Hallen

North Carolina State Univ.

Dept. of Physics

P.O. Box 8202

Raleigh, NC 27695-8202, USA

Karl Hess

Univ. of Illinois

Beckman Institute

405 North Mathews Avenue

Urbana, IL 61801-2325, USA

T. K. Higman

Univ. of Minnesota

Dept. of Electrical Engineering 200 Union St. SE

Minneapolis, MN 55455-0160

$i x$ 
D. H. Huang

Aono Atomcraft Project

Exploratory Research for Advanced

Technology

Japan Research Development Corp.

Tokodai 5-9-9

Tsukuba, Ibaraki 300-26, Japan

H. J. Hug

Univ. of Basel

Institute of Physics

Klingelbergstrassse 82

CH-4056 Basel, Switzerland

T. A. Jung

Univ. of Basel

Institute of Physics

Klingelbergstrassse 82

CH-4056 Basel, Switzerland

Dieter P. Kern

IBM Thomas J. Watson Research Ctr.

MS 17-207

P.O. Box 218

Yorktown Heights, NY 10598-0218, USA

A. Kobayashi

Aono Atomcraft Project

Exploratory Research for Advanced

Technology

Japan Research Development Corp.

Tokodai 5-9-9

Tsukuba, Ibaraki 300-26, Japan

G. P. Kochanski

AT\&T Bell Labs.

Murray Hill, NJ 07974, USA

Charles Lieber

Harvard Univ.

Applied Sciences

Dept. of Chemistry

Cambridge, MA 02138, USA

Stuart M. Lindsay

Arizona State Univ.

Dept. of Physics

Tempe, AZ 85287-0001, USA

Joseph W. Lyding

Univ. of Illinois

Dept. of Electrical and Computer

Engineering

Urbana, IL 61801, USA
Arun Majumdar

Univ. of California/Santa Barbara

Dept. of Mechanical \& Environmental

Engineering

Santa Barbara, CA 93106-5070, USA

Herschel M. Marchman

AT\&T Bell Labs.

Rm. 7E-411

600 Mountain Ave.

Murray Hill, NJ 07974-2008, USA

Christie R. K. Marrian

Naval Research Lab.

Electronics Science \& Technology Div.

Code 6864

4555 Overlook Dr.

Washington, DC 20375-5347, USA

Sterling E. McBride

Univ. of Texas/ Dallas

Erik Jonsson School of Engineering and

Computer Sciences

MP 33

P.O. Box 830688

Richardson, TX 75083-0688, USA

M. A. McCord

IBM Corp.

Semiconductor Research and

Development Ctr.

P.O. Box 218

Yorktown Heights, NY 10598, USA

A. Moser

Univ. of Basel

Institute of Physics

Klingelbergstrasse 82

CH-4056 Basel, Switzerland

L. P. Muray

Cornell Univ.

Knight Lab.

National Nanofabrication Facility

Ithaca, NY 14853, USA

Munir Nayfeh

Univ. of Illinois/Urbana-Champaign

Dept. of Physics

1110 W. Green St.

Urbana, IL 61801, USA 
R. F. W. Pease

Stanford Univ.

Solid State Electronics Lab.

MC 4055, Electrical Engineering Dept.

McCullough 204

Stanford, CA 94305-4055, USA

John N. Randall

Texas Instruments Inc.

Central Research Labs.

MS 134

13588 N. Central Expressway

Dallas, TX 75243-1108, USA

Phillip E. Russell

North Carolina State Univ.

Materials Science Dept.

P.O. Box 7916

Raleigh, NC 27695-0001, USA

Jason Schneir

National Institute of Standards and

Technology

Building 220, Room A 107

Gaithersburg, MD 20899-0001, USA

U. D. Schwarz

Univ. of Hamburg

Institute of Applied Physics

Jurginsstrasse 11

20355 Hamburg, Germany

W. F. Smith

Univ. of Texas/Austin

Physics Dept.

Austin, TX 78712-1020, USA

U. Staufer

Univ, of Basel

Institute of Physics

Kingelbergstr. 82

CH-4056 Basel, Switzerland

E. C. Teague

National Institute of Standards and

Technology

Precision Engineering Div.

Building 220, Room A117

Gaithersburg, MD 20899, USA
John R. Tucker

Univ. of Illinois

Dept. of Electrical and Computer

Engineering

Urbana, IL 61801, USA

H. Uchida

Aono Atomcraft Project

Exploratory Research for Advanced

Technology

Japan Research Development Corp.

Tokodai 5-9-9

Tsukuba, Ibaraki 300-26, Japan

Michael J. Vasile

AT\&T Bell Labs.

MS 2C-105

600 Mountain Avenue

New Providence, NJ 07974-2008, USA

Grover C. Wetsel

Univ. of Texas/Dallas

Erik Jonsson School of Engineering and

Computer Sciences

MP 33

P.O. Box 830688

Richardson, TX 75083-0688, USA

Roland Wiesendanger

Univ. of Basel

Dept. of Physics

Klingelbergstrasse 82

CH-4056 Basel, Switzerland 